Pacific

Journal of

Mathematics

\title{
CLOSED SUBSETS OF THE WEYL ALCOVE AND TQFTS
}

STEPHEN F. SAWIN

Volume $228 \quad$ No. 2

December 2006 


\title{
CLOSED SUBSETS OF THE WEYL ALCOVE AND TQFTS
}

\author{
STEPHEN F. SAWIN
}

\begin{abstract}
For an arbitrary simple Lie algebra $\mathfrak{g}$ and an arbitrary root of unity $q$, we classify the closed subsets of the Weyl alcove of the quantum group $U_{q}(\mathfrak{g})$. Here a closed subset is a set such that if any two weights in the Weyl alcove are in the set, so is any weight in the Weyl alcove which corresponds to an irreducible summand of the tensor product of a pair of representations with highest weights the two original weights. The ribbon category associated to each closed subset admits a "quotient" by a trivial subcategory as described by Bruguières and Müger, to give a modular category and a framed threemanifold invariant or a spin modular category and a spin three-manifold invariant, as proved by the author.

Most of these theories are equivalent to theories defined in Sawin, Adv. Math. 165 (2002), 1-70, but several exceptional cases represent the first nontrivial examples of theories that contain noninvertible trivial objects, making the theory much richer and more complex.
\end{abstract}

\section{Introduction}

Quantum groups, that is, quantized universal enveloping algebras of simple Lie algebras, together with their representation theory, have been the subject of much fruitful investigation, and are of interest from many perspectives, but one particularly important application is to link and three-manifold invariants. The general setting is that these quantum groups are ribbon Hopf algebras, and hence their representation theory forms a ribbon category, from which one can construct an invariant of links and more generally labeled graphs embedded in $S^{3}$ with similar properties to the original Jones polynomial. When the complex parameter $q$ on which these algebras depend is a root of unity, their representation theory satisfies the more restrictive requirements of a modular category, from which one can construct a three-manifold invariant which satisfies Atiyah's axioms for topological quantum field theory. More specifically, the set of representations spanned by the subset of the irreducible representations in the Weyl alcove, with the ordinary

MSC2000: primary 17B37; secondary 57M27.

Keywords: TQFT, quantum groups, Weyl alcove, ribbon category. 
tensor product of representations replaced by the truncated tensor product, forms a modular category.

Any subset of the Weyl alcove which spans a set of representations closed under both duality and the truncated tensor product determines a new ribbon category which is a full subcategory of the original. Of course this subcategory encodes only a subset of the link information of the original theory, and on this basis does not seem of interest. However, if this ribbon subcategory happens to be modular, there is no reason to think that the resulting three-manifold invariant is determined by the original three-manifold invariant, and in fact it is not apparent that it has any connection with the original. Thus finding closed subsets of the Weyl alcove which are modular is an important question.

In fact, the modularity requirement can be relaxed quite a bit. Müger [2000] and Bruguières [2000] have shown that under favorable circumstances which hold for the quantum group examples (e.g., the existence of a unitary structure) a ribbon category admits a kind of quotient which yields a modular category in the absence of a certain easily identifiable obstruction. In fact, even in the presence of this obstruction [Sawin 2002a] a similar process yields an invariant of spin three-manifolds. Thus a classification of the closed subsets of the Weyl alcove, together with an identification of the quotient and when the quotient yields a modular category, would give a complete summary of the invariants of three-manifolds which can be constructed out of the Weyl alcove in this fashion. The present article classifies the closed subsets of the Weyl alcove and describes the subcategory of so called degenerate objects by which one quotients.

A second reason for considering the closed subsets of the Weyl alcove is that they might plausibly correspond to quotients of the quantum group, or at least of a subalgebra. In fact in the classical case the closed subsets of the Weyl chamber correspond exactly to quotients of the simply connected groups by a subgroup of the center: i.e., there is a one-to-one correspondence between closed subsets of the Weyl chamber on the one hand and Lie groups with the given Lie algebra on the other. Thus quotients associated to closed subsets of the Weyl alcove, if they exist, might be viewed as quantum analogues of the nonsimply connected groups.

Finally, in the course of the classification we shall construct some unexpected theories at level $k=2$. Some of these theories (associated to the quantum group of type $B_{n}$ and $D_{n}$ ) appear to give new invariants and admit skein relations that suggest we might be able to compute for these theories much of what can be computed in the SU(2) (i.e., $A_{1}$ ) theory. Nevertheless, these theories exhibit novel behavior (specifically, the subcategory of degenerate objects is the representation category of a nonabelian group) worthy of further study.

The analysis of the closed subsets of the Weyl alcove was begun in [Sawin 2002b]. There closed subsets which correspond precisely to the classical closed 
subsets, i.e., to nonsimply connected Lie groups, were found and their invariants identified. These subsets correspond to Chern-Simons theories for nonsimply connected Lie groups conjectured by Dijkgraaf and Witten [1990]. A second collection of closed subsets was also identified and classified there. These subsets, associated with certain corners of the Weyl alcove, formed very simple ribbon categories (in fact the category associated to the group algebra of an abelian group, with a mildly deformed $R$-matrix) and manifold invariants depending only on homology which had been studied by Murakami, Ohtsuki and Okada [Murakami et al. 1992]. Here we complete the identification of the closed subsets of the Weyl alcove, demonstrating that the closed subsets given above are the only ones except for certain special cases at level $k=2$.

The paper is organized as follows. Section 1 introduces the needed facts about Lie algebras, quantum groups and the truncated tensor product, which can all be found in [Humphreys 1972; Kirillov 1996; Kassel 1995; Turaev 1994; Sawin 2002b]. Sections 2 and 3 give the classification of the closed subsets, and Section 4 identifies when the quotient is modular and what the resulting invariant is for the exceptional cases not discussed in [Sawin 2002b]. Finally Section 5 shows that the closure under duality condition on closed subsets is implied by the closure under tensor products. This observation is fairly independent of the rest of the paper, but is a natural question. In particular it is of great relevance when using skein theory and cabling to explore link and three-manifold invariants; see for example [Turaev and Wenzl 1993; Wenzl 1993].

\section{Quantum groups and the Weyl alcove}

Let $\mathfrak{g}$ be a simple Lie algebra and let $\left\{\alpha_{i}\right\}_{i \leq r}$ be the simple roots of $\mathfrak{g}$. The weight lattice $\Lambda$ is spanned by the fundamental weights $\left\{\lambda_{i}\right\}_{i \leq r}$ given by $\left(\lambda_{i}, \alpha_{j}\right)=$ $\delta_{i, j}\left(\alpha_{i}, \alpha_{i}\right) / 2$.

The Weyl group is denoted by $\mathfrak{W}$, and the set of weights in the fundamental Weyl chamber is called $\Lambda^{+}$(we will loosely refer to this set itself as the Weyl chamber). Half the sum of the positive roots is called $\rho$, the unique short root in the Weyl chamber is called $\beta$, and the unique long root in the Weyl chamber is called $\theta$ (in the simply laced case either will refer to the unique root in the Weyl alcove). The root $\theta$ is the highest weight of the adjoint representation of $\mathfrak{g}$. The dual Coxeter number $\check{h}$ is defined to be $(\rho, \theta)+1$, the value of the quadratic Casimir on the adjoint representation.

Let $q=e^{2 \pi i /(k+\breve{h})}$, for some natural number $k$. Recall that there is an irreducible representation $V_{\lambda}$ of the quantum group $U_{q}(\mathfrak{g})$ for each weight $\lambda$ in the Weyl alcove $\Lambda_{0}$, i.e., weights $\lambda \in \Lambda^{+}$such that $(\lambda, \theta) \leq k$. Kirillov [1996] shows that the category of representations of the quantum group which are a direct sum of 
representations in the Weyl alcove forms a semisimple ribbon $*$-category if the ordinary tensor product is replaced by the truncated tensor product, $\otimes$, which is the maximal subspace of the ordinary tensor product isomorphic to a direct sum of representations in the Weyl alcove (since we will never use the ordinary tensor product in this article, we use $\otimes$ for the truncated tensor product without confusion). Kirillov's $q$ is normalized a bit differently from what we use here, which follows the conventions of [Sawin 2002b], but because of differences in the normalization of the quantum group defining relations, the above sentence still holds, and determines the normalization. The truncated tensor product operation on the lattice of isomorphism classes of representations in the category (with direct sum as addition) forms a commutative, distributive, associative multiplication with the trivial representation $V_{0}$ acting as identity, determined by

$$
V_{\lambda} \otimes V_{\gamma} \cong \bigoplus_{\eta \in \Lambda_{0}} N_{\lambda, \gamma}^{\eta} \odot V_{\eta}
$$

where $N_{\lambda, \gamma}^{\eta}$ are nonnegative integers and $N \odot V$ indicates the direct sum of $N$ copies of the representation $V$ (or equivalently, the tensor product of $V$ with an $N$ dimensional trivial representation). In [Sawin 2006] we gave the following formula for these numbers, generalizing a result from [Andersen and Paradowski 1995]:

$$
N_{\lambda, \gamma}^{\eta}=\sum_{\sigma \in \mathfrak{W}_{0}}(-1)^{\sigma} m_{\gamma}(\lambda-\sigma(\eta))
$$

where $m_{\lambda}(\mu)$ is the dimension of the $\mu$ weight space inside the classical representation of highest weight $\lambda$ and $\mathfrak{W}_{0}$ is the quantum Weyl group, which is generated by reflection about the hyperplanes $\left\{x \mid\left(x+\rho, \alpha_{i}\right)=0\right\}$ for each simple root $\alpha_{i}$ together with $\{x \mid(x, \theta)=k+1\}$. Also

$$
C_{\lambda}=q^{(\lambda, \lambda+2 \rho) / 2}
$$

and $q \operatorname{dim}(\lambda)>0$, where $q \operatorname{dim}(\lambda)$ is the invariant of the unknot and $C_{\lambda}$ is the factor which a full twist applies to the link invariant.

For simplicity, since we will only ever need to consider representations up to isomorphism, we will confuse the (isomorphism class of the) representation $V_{\lambda}$ with the weight $\lambda$, for example writing $\lambda \otimes \gamma=\bigoplus_{\eta} N_{\lambda, \gamma}^{\eta} \odot \eta$. Caution should be used with the operations $\oplus$ and $\odot$, because the weights are elements of the weight lattice and therefore admit a lattice addition and scalar multiplication denoted by $\lambda+\gamma$ and $n \lambda$, respectively, which we will also make frequent use of. Note that $\lambda+\gamma \neq \lambda \oplus \gamma$, and $n \lambda \neq n \odot \lambda$. In particular, 0 is the additive identity in the lattice, but is the multiplicative identity for $\otimes$. We hope that the brevity of the notation outweighs this modest awkwardness. 
Theorem 0 [Sawin 2002b]. There is an injection $\ell$ from $Z(G)$, the center of the simply connected Lie group with Lie algebra $\mathfrak{g}$, to the fundamental weights of the Weyl alcove such that $z$ acts on the classical representation $V_{\gamma}$ as

$$
\exp (2 \pi i(\gamma, \ell(z))) \cdot \mathrm{id}_{\gamma} .
$$

The fundamental weights $\lambda_{i}$ in the image of $\ell$ are exactly those for which $\left(\lambda_{i}, \theta\right)=1$ and the associated root $\alpha_{i}$ is long, and are also exactly those for which there is a unique element $\tau_{i}$ of the classical Weyl group sending the standard base to the base $\left\{\alpha_{j}\right\}_{j \neq i} \cup\{-\theta\}$. If we define $\phi_{i}(\gamma)=k \lambda_{i}+\tau_{i}(\gamma)$, then $\phi_{i}$ is an isometry of the Weyl alcove and of the simplex $\left\{\lambda:\left(\lambda, \alpha_{j}\right) \geq 0\right.$ and $\left.(\lambda, \theta) \leq k\right\}$ and $\phi_{i}(\lambda \otimes \gamma)=\phi_{i}(\lambda) \otimes \gamma$ If we use $k$ also to represent the map on the weight lattice which multiplies each weight by the number $k$, then $k \ell$ is a homomorphism in the sense that $k \ell\left(z z^{\prime}\right)=$ $k \ell(z) \otimes k \ell\left(z^{\prime}\right)$. Weights in the range of $k \ell$ can be characterized as extreme points of the simplex $\left\{\lambda:\left(\lambda, \alpha_{j}\right) \geq 0\right.$ and $\left.(\lambda, \theta) \leq k\right\}$ such that a neighborhood of the weight 0 intersected with the simplex is isometric to a neighborhood of the extreme point intersected with the alcove, the isometry being given by $\phi_{i}$.

If $Z$ is a subgroup of $Z(G)$, let $\Delta_{Z}$ be the image of $Z$ under $k \ell$. The subset of the Weyl chamber consisting of weights $\gamma$ such that $Z$ acts trivially on $V_{\gamma}$ is the intersection of the Weyl chamber with a sublattice of the weight lattice, and its elements are in one-to-one correspondence with representations of the Lie group $G / Z$. The intersection $\Gamma_{Z}$ of this set with the Weyl alcove may be thought of loosely as the "Weyl alcove for quantized $G / Z$," and consists of those weights $\gamma$ in the alcove for which $(\gamma, \ell(z))$ is an integer for all $z \in \mathbb{Z}$.

A few key facts about the truncated tensor product were proven in [Sawin 2002b].

Lemma 1. For any $\sigma$ in the classical Weyl group $\mathfrak{W}$, and any weights $\gamma, \lambda$ in the Weyl alcove, if $\lambda+\sigma(\gamma)$ is in the Weyl alcove, then $\lambda \otimes \gamma$ contains $\lambda+\sigma(\gamma)$ as a summand.

Lemma 2. $\lambda \otimes \lambda^{\dagger}$ contains as a summand $\theta$ if $k \geq 2$ and $\lambda$ is not a corner (i.e. a multiple of a fundamental weight such that $(\lambda, \theta)=k$ ). In the nonsimply laced case it contains as a summand $\beta$ unless $\left(\lambda, \alpha_{i}\right)=0$ for every short simple root $\alpha_{i}$.

We say a fundamental weight $\lambda_{i}$ is long or short according to whether $\alpha_{i}$ is long or short. We say a long weight $\lambda_{i}$ is sharp or dull according to whether $\left\langle\lambda_{i}, \theta\right\rangle=1$ or not. Finally, we say that a weight $\lambda$ is a long, short, sharp or dull corner if $\langle\lambda, \theta\rangle=k$ and it is a multiple of a fundamental weight with the corresponding property. Thus for example the above lemmas tell us that $\lambda \otimes \lambda^{\dagger}$ contains $\theta$ or $\beta$ as a summand unless $\lambda$ is a dull corner. 


\section{Closed subsets}

Following [Sawin 2002b], we say that a subset $\Gamma$ of the set of representations in the Weyl alcove $\Lambda_{0}$ is closed if it is closed under duals (for every $\gamma \in \Gamma$, the weight $\gamma^{\dagger}$ of the dual representation is in $\Gamma$ ) and under the truncated tensor product (if $\lambda, \gamma \in \Gamma$ then every $\eta$ such that $N_{\lambda, \gamma}^{\eta} \neq 0$, i.e. such that $\eta$ corresponds to a direct summand of $\lambda \otimes \gamma$, is in $\Gamma$ ). We will see in Section 5 that in the quantum groups case the second condition implies the first. Such subsets correspond exactly to ribbon subcategories, and Müger [2000] showed that if they meet a certain easily checked condition they admit a quotient which is modular and gives a TQFT and three-manifold invariant. The following theorem gives a complete classification of closed subsets of the Weyl alcove. The proof encompasses this and the next section.

Theorem 1. The closed subsets of the Weyl alcove are:

(a) For any subgroup $Z \subset Z(G)$, the set $\Gamma_{Z}$.

(b) For any subgroup $Z \subset Z(G)$, the set $\Delta_{Z}$.

(c) For $k=2$,

(1) $E_{7}$ the set $\left\{0, \lambda_{6}\right\}$,

(2) $E_{7}$ with set $\left\{0, \lambda_{2}, 2 \lambda_{7}\right\}$,

(3) $E_{8}$ with set $\left\{0, \lambda_{1}\right\}$,

(4) $B_{l}$ with set $\left\{0,2 \lambda_{1}, \lambda_{j}, \lambda_{2 j}, \ldots, \lambda_{(n-1) j / 2}\right\}$ where $j>2$ and $2 l+1=n j$,

(5) $D_{l}$ with set $\left\{0,2 \lambda_{1}, \lambda_{j}, \lambda_{2 j}, \ldots, \lambda_{(n-1) j}, 2 \lambda_{l-1}, 2 \lambda_{l}\right\}$ where $j>2$ and $l=$ $n j$,

(6) $D_{l}$ with set $\left\{0,2 \lambda_{1}, \lambda_{j}, \lambda_{2 j}, \ldots, \lambda_{(n-1) j / 2}\right\}$ where $j>2$ and $2 l=n j$ for $n$ odd.

Here the $\lambda_{i}$ are the fundamental weights ordered as in [Humphreys 1972]. We will call (1)-(6) the exceptional closed subsets.

Before embarking on a proof of the theorem, some general discussion is in order. Equation (1-1) is difficult to use in general, but we will find it suffices for most of our purposes to examine it carefully only when one of the factors is $\theta$ or $\beta$. Our strategy will be to show that for any $\lambda$ which is not in one of these exceptional cases and not a sharp corner(and therefore not in the image of $k \ell$ ) an appropriate tensor product of factors of $\lambda$ and $\lambda^{\dagger}$ contains $\theta$ or $\beta$. It will be easy to see that any closed subset containing $\theta$ or $\beta$ is of the first type in the theorem. By Lemma 2, $\theta$ or $\beta$ is contained in a tensor product of copies of $\lambda$ and $\lambda^{\dagger}$ unless $\lambda$ is a long corner.

By Lemma $1, \lambda \otimes \beta$ will contain as a summand with multiplicity one every weight $\lambda+\alpha$ for which $\alpha$ is a root (or a short root in the nonsimply laced case) and $\lambda+\alpha$ is in the Weyl alcove. Thus a crucial question is this : Given any dull corner $\lambda$, for which $\alpha$ is $\lambda+\alpha$ in the Weyl alcove? Of course if $\lambda_{i}+\alpha$ is in the 
Weyl alcove for $k=\left(\theta, \lambda_{i}\right)$ (i.e. when $\lambda_{i}$ is a corner), then $n \lambda_{i}+\alpha$ is in the Weyl alcove whenever $n \lambda_{i}$ is a corner.

Below we list for each dull $\lambda_{i}$ the roots (or in the nonsimply laced case short roots) $\alpha$ such that $\lambda_{i}+\alpha$ is in the Weyl alcove for $k=\left(\lambda_{i}, \theta\right)$. The labeling of the roots and fundamental weights are as in [Humphreys 1972]. The roots are written out as a sum of simple roots and $\theta$ in such a way that each additional simple root has negative inner product with the sum up to that point (as can be checked using the Cartan matrix: see Humphreys, p. 59), confirming recursively that the sum is a root. That the root is short in the nonsimply laced case and that the entire sum has nonnegative inner product with each simple root and inner product with $\theta$ less than or equal to that of $\lambda_{i}$ can be checked from the Cartan matrix and the expansion of $\theta$ (Humphreys, p. 66).

$B_{l}$ : For $2 \leq i \leq l-1 . \lambda_{i}+\alpha_{i+1}+\alpha_{i+2}+\cdots+\alpha_{l}, \lambda_{i}-\left(\alpha_{i}+\alpha_{i+1}+\cdots+\alpha_{l}\right)$.

$C_{l}: \lambda_{l}+\alpha_{1}+\alpha_{2}+\cdots+\alpha_{l-1}, \lambda_{l}-\left(\alpha_{1}+\cdots+\alpha_{l}\right)$.

$D_{l}$ : For $2 \leq i \leq l-2 . \lambda_{i}-\left(\alpha_{i-1}+\alpha_{i}+\cdots+\alpha_{l-2}+\alpha_{l-1}+\alpha_{l}+\alpha_{l-2}+\cdots+\alpha_{i}\right)$, $\lambda_{i}+\alpha_{i+1}+\alpha_{i+2}+\cdots+\alpha_{l-2}+\alpha_{l-1}+\alpha_{l}+\alpha_{l-2}+\alpha_{l-3}+\cdots+\alpha_{i+2}$ (for the cases $i=$ $l-2$, and $i=l-3$ the last formula should read $\lambda_{l-2}+\alpha_{l-1}$ and $\lambda_{l-3}+\alpha_{l-2}+\alpha_{l-1}+\alpha_{l}$ respectively).

$E_{6}: \lambda_{2}+\alpha_{1}+\alpha_{3}+\alpha_{4}+\alpha_{5}+\alpha_{6}, \lambda_{2}-\theta . \lambda_{3}+\alpha_{1}, \lambda_{3}-\theta+\alpha_{2}+\alpha_{4}+\alpha_{5}+\alpha_{6}$, $\lambda_{4}+\alpha_{1}+\alpha_{3}, \lambda_{4}+\alpha_{5}+\alpha_{6}, \lambda_{4}-\theta+\alpha_{2} . \lambda_{5}+\alpha_{6}, \lambda_{5}-\theta+\alpha_{2}+\alpha_{4}+\alpha_{3}+\alpha_{1}$.

$E_{7}: \lambda_{1}+\alpha_{3}+\alpha_{4}+\alpha_{5}+\alpha_{6}+\alpha_{7}+\alpha_{2}+\alpha_{4}+\alpha_{5}+\alpha_{6}, \lambda_{1}-\theta . \lambda_{2}-\theta+\alpha_{1}+\alpha_{3}+\alpha_{4}+$ $\alpha_{5}+\alpha_{6}+\alpha_{7} . \lambda_{3}+\alpha_{2}+\alpha_{4}+\alpha_{5}+\alpha_{6}+\alpha_{7}, \lambda_{3}-\theta-\alpha_{1} . \lambda_{4}+\alpha_{2}, \lambda_{4}+\alpha_{5}+\alpha_{6}+\alpha_{7}$. $\lambda_{5}+\alpha_{6}+\alpha_{7}, \lambda_{5}-\theta+\alpha_{1}+\alpha_{3}+\alpha_{4}+\alpha_{2} . \lambda_{6}+\alpha_{7}, \lambda_{6}-\theta+\alpha_{1}+\alpha_{3}+\alpha_{4}+\alpha_{5}+\alpha_{4}+\alpha_{3}$.

$E_{8}: \quad \lambda_{1}-\theta+\alpha_{8}+\alpha_{7}+\alpha_{6}+\alpha_{5}+\alpha_{4}+\alpha_{3}+\alpha_{2}+\alpha_{4}+\alpha_{5}+\alpha_{6}+\alpha_{7}, \lambda_{1}-$ $\left(\alpha_{3}+\alpha_{4}+\alpha_{2}+\alpha_{5}+\alpha_{6}+\alpha_{7}+\alpha_{8}+\alpha_{4}+\alpha_{5}+\alpha_{6}+\alpha_{7}\right) . \lambda_{2}-\theta+\alpha_{1}+\alpha_{3}+$ $\alpha_{4}+\alpha_{5}+\alpha_{6}+\alpha_{7}+\alpha_{8}, \lambda_{2}-\left(\alpha_{2}+\alpha_{4}+\alpha_{3}+\alpha_{1}+\alpha_{5}+\alpha_{6}+\alpha_{4}+\alpha_{3}+\alpha_{5}+\right.$ $\left.\alpha_{4}+\alpha_{2}\right) . \lambda_{3}+\alpha_{1}, \lambda_{3}-\left(\alpha_{1}+\alpha_{3}+\alpha_{4}+\alpha_{2}+\alpha_{5}+\alpha_{4}+\alpha_{3}\right) . \lambda_{4}+\alpha_{1}+\alpha_{3}$, $\lambda_{4}+\alpha_{2} . \lambda_{5}+\alpha_{1}+\alpha_{3}+\alpha_{4}+\alpha_{2}, \lambda_{5}-\theta+\alpha_{6}+\alpha_{7}+\alpha_{8} . \lambda_{6}+\alpha_{1}+\alpha_{3}+\alpha_{4}+\alpha_{2}$, $\lambda_{6}-\theta+\alpha_{7}+\alpha_{8} . \lambda_{7}+\alpha_{1}+\alpha_{3}+\alpha_{4}+\alpha_{2}+\alpha_{5}+\alpha_{6}+\alpha_{4}+\alpha_{3}+\alpha_{5}+\alpha_{4}+\alpha_{2}$, $\lambda_{7}-\theta+\alpha_{8} \cdot \lambda_{8}+\theta-\alpha_{8}-\alpha_{7}-\alpha_{6}-\alpha_{5}-\alpha_{4}-\alpha_{3}-\alpha_{2}-\alpha_{4}-\alpha_{5}-\alpha_{6}-\alpha_{7}-\alpha_{8}$, $\lambda_{8}-\theta$.

$F_{4}: \lambda_{1}+\alpha_{2}+\alpha_{3}+\alpha_{4}+\alpha_{3}, \lambda_{1}-\left(\alpha_{1}+\alpha_{2}+\alpha_{3}\right) . \lambda_{2}+\alpha_{3}+\alpha_{4}, \lambda_{2}-\left(\alpha_{2}+\alpha_{3}\right)$.

$G_{2}: \lambda_{2}+\alpha_{1}, \lambda_{2}-\left(\alpha_{1}+\alpha_{2}\right)$.

We see from this list that for each of the nonsimply laced algebras except $B_{l}$ there is a short root $\alpha$ such that $\theta+\alpha$ is in the Weyl alcove for $k \geq 2$ (For $C_{l}$ we have $\theta=\lambda_{l}$ and $\alpha=\alpha_{1}+\alpha_{2}+\cdots+\alpha_{l-1}$; for $F_{4}, \theta=\lambda_{1}$ and $\alpha=\alpha_{2}+\alpha_{3}+\alpha_{4}+\alpha_{3}$; and for $G_{2}, \theta=\lambda_{2}$ and $\alpha=\alpha_{1}$ ). Thus by Equation (1-1) $N_{\theta, \theta}^{\theta+\alpha}$ contains a contribution 
from $\sigma=1$ since $m_{\theta}(\alpha)=1$. In order for it to contain a contribution for some other $\sigma$, that $\sigma$ would have to be a reflection about a short root $\alpha_{i}$ such that $\left(\theta+\alpha, \alpha_{i}\right)=0$ and $\alpha-\alpha_{i}$ is long. If $\alpha-\alpha_{i}$ is long then $\left(\alpha, \alpha_{i}\right)=0$. One can easily check by direct computation that there is no such $\alpha_{i}$ in any of these case. Therefore $\theta \otimes \theta$ contains $\theta+\alpha$, so $\theta \otimes \theta \otimes \theta$ contains $(\theta+\alpha) \otimes \theta$, which by Lemma 1 contains $\beta$. For $B_{l}$ when $k>2$ the same argument applies to $\theta+\beta$. When $k=2$ we will see below that a power of $\theta$ contains $2 \lambda_{l}$, which is a short corner and hence a higher power contains $\beta$. We conclude that if a closed subset of the Weyl alcove contains $\theta$, it also contains $\beta$.

Lemma 3. If a closed subset of the Weyl alcove contains $\beta$, it is of the form $\Gamma_{Z}$ for some $Z$.

Proof. Consider $\lambda$ in the root lattice and in the Weyl alcove, and choose a path in the root lattice connecting $\lambda$ to 0 such that the difference between successive points in the path is a short root. By reflecting about hyperplanes of reflection in the quantum Weyl group we can replace it by such a path crossing fewer such hyperplanes, and by induction can find such a path entirely within the alcove. Then by Lemma $1 \lambda$ is contained in $\beta^{\otimes n}$, where $n$ is the length of this path. Thus if a closed subset contains $\beta$ it contains all of the root lattice $\Lambda_{r}$.

If $\lambda, \gamma$ are in the Weyl alcove and in the same coset of $\Lambda / \Lambda_{r}$, their difference is in the root lattice, and thus any closed subset containing one of these and $\beta$ contains the other. So any closed subset containing $\beta$ is a union of cosets intersected with the Weyl alcove. The tensor product of two weights is a nonempty sum (because the quantum dimensions are nonzero) and is in the product of their cosets, so the set of cosets making up such a closed subset is a subgroup of $\Lambda / \Lambda_{r}$. This proves that the closed subset is in the intersection of the preimage of a subgroup of $\Lambda / \Lambda_{r}$ with the Weyl alcove. By Theorem 0 the map sending $z \in Z(G)$ to $\exp (2 \pi i(\ell(z), \cdot)) \in$ $\left(\Lambda / \Lambda_{r}\right)^{*}$ is a group isomorphism. So such a subgroup is dual to some subgroup $Z \subset Z(G)$, and the closed subset is exactly $\Gamma_{Z}$.

Now if $\lambda$ is a dull corner it is a multiple of one of the weights in the chart above. If $\lambda_{i}+\alpha$ is in the Weyl alcove for $k=\left(\lambda_{i}, \theta\right)$, then $n \lambda_{i}+\alpha$ is in the Weyl alcove for $k=n\left(\lambda_{i}, \theta\right)$. So from the chart there are at least two elements of the Weyl alcove a short root away from $\lambda$, except if $\lambda$ is a multiple of $\lambda_{2}$ for $E_{7}$. In this exceptional case $\lambda_{2} / 2-\alpha_{2}$ is in the Weyl alcove, so there are still at least two elements of the alcove a short root away from $\lambda$ except when $\lambda=\lambda_{2}$ and $k=2$. Thus with this exception if $\lambda$ is a dull corner then by Lemma $1 \lambda \otimes \beta$ contains two weights. But each of these weights when tensored with $\beta$ contain $\lambda$, so $\lambda \otimes \beta \otimes \beta$ contains $\lambda$ with multiplicity at least 2 . Therefore $\lambda \otimes \lambda^{\dagger}$ contains two weights in $\beta \otimes \beta$. Of course one is the trivial weight, so the other must be of the form $\beta+\alpha$ for $\alpha$ a short root 
different from $-\beta$. If this $\beta+\alpha$ is not a corner dual to a long root, a tensor power of it then contains $\beta$.

\section{Proof of the Theorem}

Proof of Theorem 1. It was shown in [Sawin 2002b] that the subsets of the form $\Gamma_{Z}$ and $\Delta_{Z}$ are closed. We will see below that the exceptional cases are closed by computing the truncated tensor product completely. So we have only to show that every closed subset is of this form.

We will assume the closed set contains some $\lambda$ not in the image of $k \ell$, and show it is either the exceptional case or it contains $\theta$ or $\beta$, in which case by Lemma 3 it falls into the first category. By Lemma 2 we may assume $\lambda$ is a corner dual to a long root.

Case $\boldsymbol{k}=1$. There are no dull corners, so there is nothing to prove.

Case $\boldsymbol{k}=2$. Except for $\lambda_{2}$ of $E_{7}$ if $\lambda$ is a corner dual to a long root and not in the range of $k \ell$ then $\lambda \otimes \lambda^{\dagger}$ contains something nontrivial in $\beta \otimes \beta$, so we may assume that $\lambda$ is a such a corner and $\lambda \otimes \lambda^{\dagger}$ contains a summand of the form $\beta+\alpha$ for $\alpha$ a short root.

- For $A_{l}$, there are no dull corners.

- For $B_{l}$, there is nothing to prove if $l=2$, so assume $l>2$. The Weyl alcove consists of $\lambda_{i}$ for $i \leq l$ and $2 \lambda_{1}$ and $2 \lambda_{l}$. By checking which differences among these are short roots and noting $\lambda_{1}=\beta$ we conclude

$$
\begin{aligned}
\lambda_{i} \otimes \lambda_{1} & = \begin{cases}\lambda_{i-1} \oplus \lambda_{i+1} & \text { for } 1<i<l-1, \\
0 \oplus 2 \lambda_{1} \oplus \lambda_{2} & \text { for } i=1, \\
2 \lambda_{l} \oplus \lambda_{l-2} & \text { for } i=l-1, \\
\lambda_{l} & \text { for } i=l,\end{cases} \\
2 \lambda_{l} \otimes \lambda_{1} & =2 \lambda_{l} \oplus \lambda_{l-1}, \\
2 \lambda_{1} \otimes \lambda_{1} & =\lambda_{1}, \\
2 \lambda_{1} \otimes 2 \lambda_{1} & =0 .
\end{aligned}
$$

From this we conclude recursively that

$$
\begin{aligned}
2 \lambda_{1} \otimes \lambda_{i} & =\lambda_{i} \quad \text { for } i \leq l, \\
2 \lambda_{1} \otimes 2 \lambda_{l} & =2 \lambda_{l},
\end{aligned}
$$




$$
\lambda_{i} \otimes \lambda_{j}= \begin{cases}\lambda_{i-j} \oplus \lambda_{i+j} & \text { for } l>i>j \text { and } i+j<l \\ \lambda_{i-j} \oplus 2 \lambda_{l} & \text { for } l>i>j \text { and } i+j=l, l+1 \\ \lambda_{i-j} \oplus \lambda_{2 l+1-i-j} & \text { for } l>i>j \text { and } i+j>l+1 \\ 0 \oplus 2 \lambda_{1} \oplus \lambda_{2 i} & \text { for } l>i=j \text { and } 2 i<l \\ 0 \oplus 2 \lambda_{1} \oplus 2 \lambda_{l} & \text { for } l>i=j \text { and } 2 i=l, l+1 \\ 0 \oplus 2 \lambda_{1} \oplus \lambda_{2 l+1-2 i} & \text { for } l>i=j \text { and } 2 i>l+1 .\end{cases}
$$

Notice first of all that a closed subset containing $\lambda_{2}=\theta$ will contain $\lambda_{i}$ for all even $i<l$. In particular it must contain either $\lambda_{l-2}$ or $\lambda_{l-1}$, so it must contain $2 \lambda_{l}$, and therefore since this is a short corner it must contain $\beta$. Thus we recover our promised assertion that even for $B_{l}$ at level $k=2$, a closed subset containing $\theta$ contains $\beta$ and therefore is of the from $\Gamma_{Z}$.

Let $\Gamma$ be a closed subset which is not of the form $\Gamma_{Z}$ or $\Delta_{Z}$. We know that $\Gamma$ cannot contain $\lambda_{1}, \lambda_{2}, \lambda_{l}$ or $2 \lambda_{l}$, or else it would be of the form $\Gamma_{Z}$, and $\Gamma$ must contain something other than 0 and $2 \lambda_{1}$. So let $j$ be the least $j$ such that $\lambda_{j} \in \Gamma$. Necessarily $l>j>2$. By the product rules above $\lambda_{j}, \lambda_{2 j}, \lambda_{3 j}, \ldots \in \Gamma_{Z}$. Suppose $m$ is the largest such that $\lambda_{m j} \in \Gamma$. Then every summand of $\lambda_{m j} \otimes \lambda_{j}$ is in $\Gamma$. Clearly $(m+1) j \geq l$, and in fact $(m+1) j>l+1$, or else $2 \lambda_{l} \in \Gamma$. So we conclude that $\lambda_{2 l+1-(m+1) j} \in \Gamma$. Now $m j<l<(m+1) j$ so $2 l+1-(m+1) j$ is within $j$ of $m j$. If they are not equal then $\lambda_{m j} \otimes \lambda_{2 l+1-(m+1) j}$ contains $\lambda_{i}$ where $i$ is this difference, contradicting the minimality of $j$. Thus we conclude $m j=2 l+1-(m+1) j$, and $\Gamma$ contains set (4) in the statement of the theorem, with $n=2 m+1$. If it contained any $\lambda_{i}$ not in this set, there would be $p$ such that $|i-p j|<j$, and hence $\lambda_{|i-p j|}$ would be in the set, contradicting the minimality of $j$.

- For $C_{l}$ there is nothing to prove since $\theta$ is the only dull corner.

- For $D_{l}$ at $k=2$ the weights are $\lambda_{i}$ for $1 \leq i \leq l, 2 \lambda_{1}, 2 \lambda_{l-1}, 2 \lambda_{l}$, and $\lambda_{l-1}+\lambda_{l}$. By checking which differences among these are roots we see

$$
\lambda_{i} \otimes \theta= \begin{cases}\lambda_{1} \oplus \lambda_{3} & \text { for } i=1 \\ 0 \oplus 2 \lambda_{1} \oplus \lambda_{4} & \text { for } i=2 \\ \lambda_{i-2} \oplus \lambda_{i+2} & \text { for } 2<i<l-3 \\ \lambda_{l-5} \oplus\left(\lambda_{l-1}+\lambda_{l}\right) & \text { for } i=l-3 \\ \lambda_{l-4} \oplus 2 \lambda_{l} \oplus 2 \lambda_{l-1} & \text { for } i=l-2 .\end{cases}
$$

Since $2 \lambda_{1}$ is in the range of $k \ell$ and hence invertible, it follows $2 \lambda_{1} \otimes \theta=\theta$. Since $\lambda_{1} \otimes \lambda_{1}$ contains $2 \lambda_{1}$ by Lemma 1 , we conclude $2 \lambda_{1} \otimes \lambda_{1}=\lambda_{1}$ so inductively 
$2 \lambda_{1} \otimes \lambda_{i}=\lambda_{i}$ for $i<l-1$. Similarly

$$
\begin{aligned}
2 \lambda_{l} \otimes \theta & =\lambda_{l-2}, \\
2 \lambda_{l-1} \otimes \theta & =\lambda_{l-2}, \\
2 \lambda_{l} \otimes 2 \lambda_{1} & =2 \lambda_{l-1}, \\
2 \lambda_{l-1} \otimes 2 \lambda_{1} & =2 \lambda_{l} .
\end{aligned}
$$

Since $\lambda_{1} \otimes \theta$ contains $\lambda_{1}$, it is clear that $\lambda_{1} \otimes \lambda_{1}$ contains $0, \theta$, and $2 \lambda_{1}$, each with multiplicity one. Notice that by Equation (1-1), $\eta$ is not a direct summand of $\lambda \otimes \gamma$ if the distance between $\lambda$ and $\eta$ is more than $\|\gamma\|$ (this is argued explicitly in the proof of [Sawin 2002b, Lemma 2]). Noting that $\left\|\lambda_{1}\right\|=1$ and computing $\left\|\lambda-\lambda_{1}\right\|$ for $\lambda=\lambda_{i}$ with $2<i<l-1$ and $\lambda=\lambda_{l-1}, \lambda_{l},\left(\lambda_{l-1}+\lambda_{l}\right)$ we conclude

$$
\lambda_{1} \otimes \lambda_{1}=0 \oplus \theta \oplus 2 \lambda_{1} .
$$

It then follows recursively that

$$
\begin{aligned}
\lambda_{i} \otimes \lambda_{1} & = \begin{cases}\lambda_{i-1} \oplus \lambda_{i+1} & \text { for } 1<i<l-2 \\
\lambda_{l-3} \oplus\left(\lambda_{l-1}+\lambda_{l}\right) & \text { for } i=l-2,\end{cases} \\
\left(\lambda_{l-1}+\lambda_{l}\right) \otimes \lambda_{1} & =\lambda_{l-2} \oplus 2 \lambda_{l-1} \oplus 2 \lambda_{l}, \\
2 \lambda_{l-1} \otimes \lambda_{1} & =\lambda_{l-1}+\lambda_{l}, \\
2 \lambda_{l} \otimes \lambda_{1} & =\lambda_{l-1}+\lambda_{l} .
\end{aligned}
$$

Finally, we get recursively from this

$$
\begin{aligned}
& \lambda_{i} \otimes \lambda_{j}= \begin{cases}\lambda_{i-j} \oplus \lambda_{i+j} & \text { for } j<i<l-1 \text { and } i+j<l-1 \\
\lambda_{i-j} \oplus\left(\lambda_{l-1}+\lambda_{l}\right) & \text { for } j<i<l-1 \text { and } i+j=l-1, l+1 \\
\lambda_{i-j} \oplus 2 \lambda_{l-1} \oplus 2 \lambda_{l} & \text { for } j<i<l-1 \text { and } i+j=l \\
\lambda_{i-j} \oplus \lambda_{2 l-i-j} & \text { for } l>i>j \text { and } i+j>l+1 \\
0 \oplus 2 \lambda_{1} \oplus \lambda_{i+j} & \text { for } j=i<l-1 \text { and } i+j<l-1 \\
0 \oplus 2 \lambda_{1} \oplus\left(\lambda_{l-1}+\lambda_{l}\right) & \text { for } j=i<l-1 \text { and } 2 i=l-1, l+1 \\
0 \oplus 2 \lambda_{1} \oplus 2 \lambda_{l-1} \oplus 2 \lambda_{l} & \text { for } j=i<l-1 \text { and } 2 i=l \\
0 \oplus 2 \lambda_{1} \oplus \lambda_{2 l-2 i} & \text { for } j=i<l \text { and } 2 i>l+1,\end{cases} \\
& \lambda \otimes \lambda_{j}= \begin{cases}\lambda_{l-1}+\lambda_{l} & \text { for } \lambda=2 \lambda_{l-1} \text { or } \lambda=2 \lambda_{l} \text { and } j=1 \\
\lambda_{l-j} & \text { for } \lambda=2 \lambda_{l-1} \text { or } \lambda=2 \lambda_{l} \text { and } 1<j<l-1 .\end{cases}
\end{aligned}
$$


Thus if the closed subset $\Gamma$ contains $\lambda_{1}, \lambda_{2}, \lambda_{l-1}, \lambda_{l}$ or $\lambda_{l-1}+\lambda_{l}$ it contains $\theta$ and is of the form $\Gamma_{Z}$. If it contains only a subset of $0,2 \lambda_{1}, 2 \lambda_{l-1}$, and $2 \lambda_{l}$ it is of the form $\Delta_{Z}$. If $\Gamma$ is not of the form $\Gamma_{Z}$ or $\Delta_{Z}$ then it must contain $\lambda_{j}$ for some $2<j<l-1$, so suppose $j$ is the least such. Then $\Gamma$ contains $\lambda_{j}, \lambda_{2 j}, \ldots, \lambda_{m j}$, where $m$ is the greatest such that $m j<l-1$. Again $\Gamma$ must contain every summand of $\lambda_{m j} \otimes \lambda_{j}$. Then by the maximality of $m$ we know $(m+1) j>l-2$, and since $\Gamma$ cannot contain $\lambda_{l-1}+\lambda_{l}$ we know $(m+1) j \neq l-1, l+1$. If $(m+1) j=l$, then $\Gamma$ contains $\left\{0,2 \lambda_{1}, \lambda_{j}, \lambda_{2 j}, \ldots, \lambda_{m j}, 2 \lambda_{l-1}, 2 \lambda_{l}\right\}$. If it contained any other $\lambda_{i}$ for $2<i<l-1$ then there would be $p$ with $|i-p j|<j$, so $\lambda_{|i-p j|}$ would be in the set, contradicting the minimality of $j$. Since $\Gamma$ cannot contain any other weights in the alcove, we conclude $\Gamma$ is of the form of set (5) in the theorem, with $m=n-1$.

On the other hand if $(m+1) j \neq l$, the $(m+1) j>l+1$, and therefore $\lambda_{2 l+1-(m+1) j}$. Of course $2 l+1-(m+1) j$ is a distance less than $j$ from $m j$, so if the difference is nonzero then again we contradict the minimality of $j$. Therefore the distance between them is zero, so $2 l+1=(2 m+1) j$, and $\Gamma$ contains $\left\{0,2 \lambda_{1}, \lambda_{j}, \cdots, \lambda_{m j}\right\}$. Again it cannot contain any other weight in the Weyl alcove without contradicting the minimality of $j$ (if it contained $2 \lambda_{l}$ or $2 \lambda_{l-1}$ it would contain $\lambda_{l-j}$, which is distinct from $\lambda_{m j}$ but $l-j$ is less than $j$ away from $m j$ ) so $\Gamma$ is set (6) in the theorem, with $n=2 m+1$.

- For $E_{6}$, the weights of the Weyl alcove are $0, \lambda_{1}, 2 \lambda_{1}, \lambda_{2}, \lambda_{3}, \lambda_{5}, \lambda_{6}, 2 \lambda_{6}$, and $\lambda_{1}+\lambda_{6}$. A closed subset containing a dull corner must contain a nontrivial weight of the form $\theta+\alpha$, but the only such weight is $\lambda_{1}+\lambda_{6}$, which is not a corner.

- For $E_{7}$, the weights in the alcove are those in $k \ell$ ( 0 and $\left.2 \lambda_{7}\right)$, the other corners $\left(\lambda_{1}=\theta, \lambda_{2}\right.$ and $\left.\lambda_{6}\right)$ and one other $\left(\lambda_{7}\right)$. We have

$$
\begin{aligned}
2 \lambda_{7} \otimes \theta & =\lambda_{6}, \\
\theta \otimes \theta & =0 \oplus \lambda_{6}, \\
\lambda_{6} \otimes \theta & =\theta \oplus 2 \lambda_{7}, \\
\lambda_{2} \otimes \theta & =\lambda_{7}, \\
\lambda_{7} \otimes \theta & =\lambda_{2} \oplus \lambda_{7},
\end{aligned}
$$

so

$$
\lambda_{6} \otimes \lambda_{6}=0 \oplus \lambda_{6}, \quad \lambda_{2} \otimes \lambda_{6}=\lambda_{7}, \quad \lambda_{7} \otimes \lambda_{6}=\lambda_{2} \oplus \lambda_{7} .
$$

Since every weight in $E_{7}$ is self-dual, $\lambda_{7} \otimes \lambda_{7}$ consists of weights in the root lattice, and since $N_{\lambda, \gamma}^{\delta}=N_{\lambda, \delta^{*}}^{\gamma^{*}}$ we can read off from the previous equations

$$
\lambda_{7} \otimes \lambda_{7}=0 \oplus \theta \oplus 2 \lambda_{7} \oplus \lambda_{6},
$$

So

$$
\lambda_{2} \otimes \lambda_{7}=\theta \oplus \lambda_{6} \quad \text { and } \quad \lambda_{2} \otimes \lambda_{2}=0 \oplus 2 \lambda_{7}
$$


Thus the smallest closed subset containing $\lambda_{6}$ is $\left\{0, \lambda_{6}\right\}$, the smallest one containing $\lambda_{2}$ is $\left\{0, \lambda_{2}, 2 \lambda_{7}\right\}$, and the smallest one containing any other nonunit is a subset containing $\theta$.

- For $E_{8}$, the only weights are $0, \lambda_{1}$ and $\lambda_{8}=\theta$. As above we see that $\theta \otimes \theta=0 \oplus \lambda_{1}$, $\lambda_{1} \otimes \theta=\theta$, and hence $\lambda_{1} \otimes \lambda_{1}=0$. Thus $\lambda_{1}$ is invertible, $\left\{\lambda_{1}, 0\right\}$ is a closed subset, and every other closed subset contains $\theta$.

- For $F_{4}$ and $G_{2}$ there is nothing to prove since the only elements are $0, \theta, \beta$, and $2 \beta$, where $2 \beta$ is a short weight.

Case $\boldsymbol{k}=3$. Since now for all dull corners we know $\lambda \otimes \lambda^{\dagger}$ contains something nontrivial in $\beta \otimes \beta$, we may assume our closed set contains a corner which is of the form $\beta+\alpha$ for $\alpha$ short.

In the nonsimply laced case there are no such corners, so we need only consider the simply laced case. The only corners are fundamental weights with $\left(\lambda_{i}, \theta\right)=3$ together with the range of $k \ell$.

- For $D_{l}$ there are no fundamental weights with $\left(\lambda_{i}, \theta\right)=3$, and the range of $k \ell$, $\left(3 \lambda_{1}, 3 \lambda_{l-1}\right.$, and $\left.3 \lambda_{l}\right)$ contains nothing in the root lattice.

- For $E_{6}$, neither $3 \lambda_{1}$ nor $3 \lambda_{6}$ is the sum of two roots, so only $\lambda_{4}$ is such a corner. Since $\lambda_{4} \otimes \theta$ contains at least three summands, $\lambda_{4} \otimes \lambda_{4}$ contains two distinct nontrivial summands of $\theta \otimes \theta$, so it must contain a noncorner.

- For $E_{7}$, since $3 \lambda_{7}$ is not in the root lattice, only $\lambda_{3}$ and $\lambda_{5}$ are such corners, and only $\lambda_{3}$ among all corners is of the form $\theta+\alpha$. Thus any closed subset containing a corner contains $\lambda_{3}$, so since $\lambda_{3} \otimes \theta$ contains at least three summands, $\lambda_{3} \otimes \lambda_{3}$ contains two distinct nontrivial summands of $\theta \otimes \theta$, one of which must not be a corner.

- For $E_{8}$, Only $\lambda_{2}$ and $\lambda_{7}$ are corners, and neither is of the form $\theta+\alpha$.

Case $\boldsymbol{k}=4$. Again we need consider only the simply laced case, and we need only consider corners of the form $\theta+\alpha$, which means the corner $2 \theta$. Now in each case $\theta=\lambda_{i}$ for some $i$, so in addition to the weights in the chart, we have $2 \theta-\alpha_{i}$ is a summand of $2 \theta \otimes \theta$, so $2 \theta \otimes 2 \theta$ contains two distinct nontrivial summands of $\theta \otimes \theta$, one of which must not be a corner.

Case $\boldsymbol{k}>$ 4. For any dull corner $\lambda, \lambda \otimes \lambda^{*}$ contains a nontrivial summand of $\theta \otimes \theta$, which cannot be a corner. 


\section{Modular categories and closed subsets of the Weyl alcove}

As alluded to in the introduction, there is a well-defined procedure for constructing a modular or spin modular category out a ribbon $*$-category (all of our ribbon categories inherit the $*$-structure from the original ribbon category). These techniques were developed by Müger [2000] and Bruguières [2000].

First one should identify the subcategory of degenerate objects, which are simple objects (in our case irreducible representations) $\lambda$ such that $R_{\lambda, \gamma}=R_{\gamma, \lambda}^{-1}$ for every simple object $\gamma$ in the ribbon category, where $R$ is the $R$-morphism associated to a crossing. These come in two sorts, even or odd, according to whether the effect of the full twist $C_{\lambda}$ is multiplication by one or minus one. If all are even, one can quotient by them to get a modular category. If in addition they are all invertible and form a cyclic group, [Sawin 2002a] offers a detailed description of the invariant and TQFT in terms of the original category. In the same work it is shown that if there are odd degenerate objects, one can still quotient by the even degenerate objects and the result is a spin-modular category which gives an invariant of spin three-manifolds.

For the ribbon categories associated to the closed sets of the form $\Gamma_{Z}$ and $\Delta_{Z}$ [Sawin 2002b] proves that all degenerate objects are invertible and identifies in which cases they are all odd. Below we identify for each exceptional closed category what the degenerate objects are, when they are even, when they are invertible, and when the resulting TQFT is equivalent to a nonexceptional TQFT.

The case $E_{7}$ with $\left\{0, \lambda_{6}\right\}$. Here $\lambda_{6} \otimes \lambda_{6}=0 \oplus \lambda_{6}$.

For $E_{7}$ we have $h=18$ so $k+h=20$. We have $\left(\lambda_{6}, \lambda_{6}\right)=4$ and $\left(\lambda_{6}, \rho\right)=26$, so

$$
C_{\lambda_{6}}=e^{2 \pi i(4+2 \cdot 26) /(2 \cdot 20)}=e^{4 \pi i / 5} .
$$

Since this is not \pm 1 then $\lambda_{6}$ is not degenerate, so the set is in itself modular.

Straightforward calculations show that this ribbon category is determined up to isomorphism by the link invariant, which is in turn determined by a skein relation, the same skein relation that determines the $\mathrm{SO}(3)$ theory at $k=3$, and thus this ribbon category is isomorphic to the $\mathrm{SO}(3)$ level 3 category.

The case $\boldsymbol{E}_{7}$ with $\left\{\mathbf{0}, \lambda_{2}, \mathbf{2} \lambda_{7}\right\}$. Here $\lambda_{2} \otimes \lambda_{2}=0 \oplus 2 \lambda_{7}$ and $\lambda_{2} \otimes 2 \lambda_{7}=\lambda_{2}$.

Again $h+k=20,\left(\lambda_{2}, \lambda_{2}\right)=7 / 2,\left(\lambda_{2}, \rho\right)=49 / 2,\left(2 \lambda_{7}, 2 \lambda_{7}\right)=6$ and $\left(2 \lambda_{7}, \rho\right)=$ 27 so

$$
\begin{aligned}
C_{\lambda_{2}} & =e^{2 \pi i(7 / 2+2 \cdot 49 / 2) /(2 \cdot 20)}=e^{5 \pi i / 8} \\
C_{2 \lambda_{7}} & =e^{2 \pi i(6+2 \cdot 27) /(2 \cdot 20)}=-1 .
\end{aligned}
$$

Of course

$$
R_{\lambda_{2}, 2 \lambda_{7}} R_{2 \lambda_{7}, \lambda_{2}}=C_{\lambda_{2}} C_{\lambda_{2}}^{-1} C_{2 \lambda_{7}}^{-1}=-1
$$


so neither $2 \lambda_{7}$ nor $\lambda_{2}$ is degenerate and the theory is modular. Again direct calculation shows that this theory is isomorphic to the SU(2) theory at level $k=2$ but this time with a nonstandard choice for $q^{1 / 4}=e^{13 \pi i / 8}$ (see [Sawin 2006]).

The case $E_{8}$ with $\left\{0, \lambda_{1}\right\}$. Here $\lambda_{1} \otimes \lambda_{1}=0$.

For $E_{8} h=30$ so $k+h=32$. $\left(\lambda_{1}, \lambda_{1}\right)=4$ and $\left(\lambda_{1}, \rho\right)=46$ so

$$
C_{\lambda_{1}}=e^{2 \pi i(4+2 \cdot 46) /(2 \cdot 32)}=-1 .
$$

This is an odd degenerate object and of course the ribbon category is isomorphic to that for $\mathrm{SO}(3)$ at $k=2$. As argued in [Sawin 2002a], this gives the trivial invariant of spin three-manifolds.

The case $B_{l}$ with $\left\{0,2 \lambda_{1}, \lambda_{j}, \lambda_{2 j}, \ldots, \lambda_{(n-1) j / 2}\right\}$ where $2 l+1=n j$. Here $h=$ $2 l-1$ so $k+h=2 l+1$. Also, $\left(\rho, \lambda_{i}\right)=l i-i^{2} / 2$ and $\left(\lambda_{i}, \lambda_{i}\right)=i$. Thus

$$
C_{2 \lambda_{1}}=e^{\pi i(4+2(2 l-1)) /(2 l+1)}=1
$$

and thus

$$
R_{2 \lambda_{1}, \lambda_{m j}} R_{\lambda_{m j}, 2 \lambda_{1}}^{-1}=1,
$$

so $2 \lambda_{1}$ is even degenerate. On the other hand

$$
C_{\lambda_{m j}}=e^{\pi i\left(m j-m^{2} j^{2} /(2 l+1)\right)},
$$

so

$$
\begin{aligned}
C_{\lambda_{(m \pm p) j}} C_{\lambda_{m j}}^{-1} C_{\lambda_{p j}}^{-1} & =e^{\mp 2 \pi i m p j^{2} /(2 l+1)}, \\
C_{\lambda_{2 l+1-(m+p) j}} C_{\lambda_{m j}}^{-1} C_{\lambda_{p j}}^{-1} & =e^{-2 \pi i m p j^{2} /(2 l+1)}, \\
C_{2 \lambda_{1}} C_{\lambda_{m j}}^{-1} C_{\lambda_{m j}}^{-1}=C_{0} C_{\lambda_{m j}}^{-1} C_{\lambda_{m j}}^{-1} & =e^{2 \pi i m^{2} j^{2} /(2 l+1)} .
\end{aligned}
$$

This will be 1 for all $p$, and thus $\lambda_{m j}$ will be degenerate, exactly if $m$ is a multiple of $n / d$, where $d$ is the greatest common divisor of $n$ and $j$. If $m=r n / d$ then

$$
C_{\lambda_{m j}}=e^{\pi i\left(m j-m^{2} j^{2} /(2 l+1)\right)}=e^{\pi i\left(r(2 l+1) / d-r^{2}(2 l+1) / d^{2}\right)} .
$$

Since $(2 l+1) / d$ and $(2 l+1) / d^{2}$ are both odd, this is one whether $r$ is even or odd, and thus all such $\lambda_{m j}$ are even. Thus we get a modular category which is a quotient by the $\mathbb{Z} / 2$ action if $m$ and $j$ are relatively prime, and by the set of even simple degenerates

$$
\left\{0,2 \lambda_{1}, \lambda_{(2 l+1) / d}, \lambda_{2(2 l+1) / d}, \ldots, \lambda_{(d-1)(2 l+1) /(2 d)}\right\}
$$

if $(n, j)=d \neq 1$. Notice that when $d \neq 1$ the set of simple degenerates does not form a group, which is to say that there are noninvertible degenerate objects. In fact one can check that the subcategory generated by these representations 
is isomorphic to the representation theory of the nonabelian group presented by $\left\langle x, y \mid x^{2}=y^{2}=(x y)^{d}=1\right\rangle$ (any eigenvector of $x y$ generates a two-dimensional irreducible subrepresentation if the eigenvalue of $x y$ is a nontrivial $d$-th root of unity and a one-dimensional subrepresentation if the eigenvalue is 1 , recalling that $d$ is necessarily odd. These two-dimensional representations are classified by the eigenvalue of $x y$, and the one-dimensional by the eigenvalue of $x$.) For example, when $l=13$ and $j=3$, we have $n=9, d=3$, and the subcategory generated by

$$
\left\{0,2 \lambda_{1}, \lambda_{3}, \lambda_{6}, \lambda_{9}, \lambda_{12}\right\}
$$

contains as its trivial subcategory

$$
\left\{0,2 \lambda_{1}, \lambda_{9}\right\}
$$

where $2 \lambda_{2} \otimes 2 \lambda_{1}=0,2 \lambda_{1} \otimes \lambda_{9}=\lambda_{9}$, and $\lambda_{9} \otimes \lambda_{9}=0 \oplus 2 \lambda_{1} \oplus \lambda_{9}$.

This is the first example of which the author is aware of a nonsymmetric ribbon category with noninvertible degenerate objects. Whether the resulting quotient gives a truly new TQFT is not clear, and in any case this example is worthy of further study.

The case $D_{l}$ with $\left\{0,2 \lambda_{1}, \lambda_{j}, \lambda_{2 j}, \ldots, \lambda_{(n-1) j}, 2 \lambda_{l-1}, 2 \lambda_{l}\right\}$ where $l=n j$. Here $h=2 l-2$ so $k+h=2 l,\left(\rho, \lambda_{i}\right)=i(2 l-i-1) / 2$, and $\left(\lambda_{i}, \lambda_{i}\right)=i$, so

$$
\begin{aligned}
C_{2 \lambda_{1}} & =e^{\pi i(4+2 \cdot 2 \cdot(2 l-2) / 2) /(2 l)}=1, \\
C_{2 \lambda_{l}} & =e^{\pi i(4 l+2 \cdot 2 l(2 l-l-1) / 2) /(2 l)}=e^{\pi i(l+1)}, \\
C_{2 \lambda_{l-1}} & =e^{\pi i(4(l-1)+2 \cdot 2(l-1)(2 l-l) / 2) /(2 l)}=e^{\pi i(l+1)}, \\
C_{\lambda_{m j}} & =e^{\pi i(m j+2 m j(2 l-m j-1) / 2) /(2 l)}=e^{\pi i\left(m j-m^{2} j^{2} /(2 l)\right)},
\end{aligned}
$$

so $2 \lambda_{1}$ is even degenerate and

$$
\begin{aligned}
R_{2 \lambda_{l}, \lambda_{m j}} R_{\lambda_{m j}, 2 \lambda_{l}}^{-1} & =C_{\lambda_{l-m j}} C_{\lambda_{m j}}^{-1} C_{2 \lambda_{l}}^{-1}=e^{\pi i(-l / 2-m j-1)}, \\
R_{2 \lambda_{l-1} l, \lambda_{m j}} R_{\lambda_{m j}, 2 \lambda_{l-1}}^{-1} & =C_{\lambda_{l-m j}} C_{\lambda_{m j}}^{-1} C_{2 \lambda_{l-1}}^{-1}=e^{\pi i(-l / 2-m j-1)},
\end{aligned}
$$

and $2 \lambda_{l}, 2 \lambda_{l-1}$ are thus degenerate exactly when $j$ is even and $l / 2$ is odd. They are always odd degenerate. Also

$$
\begin{gathered}
C_{\lambda_{(m \pm p) j}} C_{\lambda_{p j}}^{-1} C_{\lambda_{m j}}^{-1}=e^{\mp \pi i m p j^{2} / l}, \\
C_{\lambda_{l-(m+p) j}} C_{\lambda_{m j}}^{-1} C_{\lambda_{p j}}^{-1}=e^{-\pi i m p j^{2} / l}, \\
C_{2 \lambda_{1}} C_{\lambda_{m j}}^{-1} C_{\lambda_{m j}}^{-1}=C_{0} C_{\lambda_{m j}}^{-1} C_{\lambda_{m j}}^{-1}=e^{-\pi i m^{2} j^{2} / l}, \\
C_{2 \lambda_{l-1}} C_{\lambda_{m j}}^{-1} C_{\lambda_{l-m j}}^{-1}=C_{2 \lambda_{l}} C_{\lambda_{m j}}^{-1} C_{\lambda_{l-m j}}^{-1}=e^{\pi i\left(1+m j^{2} / l-m j+l / 2\right)} .
\end{gathered}
$$


This will be 1 for all $p$, and thus $\lambda_{m j}$ will be degenerate, exactly when $l$ is even, $l / 2$ is odd, and either $m$ is an even multiple of $n / d$, where $d$ is the greatest common divisor of $n$ and $j$, or $j$ is even and $m$ is a multiple of $l / d$. If $m=r n / d$, then

$$
C_{\lambda_{m j}}=e^{\pi i\left(r l / d-r^{2} l /\left(2 d^{2}\right)\right)}
$$

which is 1 if and only if $r$ is even.

Thus the set of simple degenerate elements consists of

$$
\left\{0,2 \lambda_{1}\right\}
$$

if (a) $l$ is odd, or (b) $l$ and $l / 2$ are even, or (c) $l$ is even, $l / 2$ is odd and $\operatorname{gcd}(n, j)=1$. In this case the quotient is modular.

The set of simple degenerate objects consists of

$$
\left\{0,2 \lambda_{1}, \lambda_{2 l / d}, \lambda_{4 l / d}, \ldots, \lambda_{(d-1) l / d}\right\}
$$

if $l$ is even, $j$ and $l / 2$ are odd and $\operatorname{gcd}(n, j) \neq 1$. In this case all the degenerate objects are even and the quotient is modular. The subcategory of degenerate objects is isomorphic to the category of representations of the group $\langle x, y| x^{2}=y^{2}=$ $\left.(x y)^{(d+1) / 2}=1\right\rangle$.

The set of simple degenerate objects is

$$
\left\{0,2 \lambda_{1}, \lambda_{l / d}, \lambda_{2 l / d}, \ldots, \lambda_{(d-1) l / d}, 2 \lambda_{l-1}, 2 \lambda_{l}\right\}
$$

if $l$ and $j$ are even and $l / 2$ is odd (here the set is understood to have just four elements if $d=1$ ). In this case the odd multiples of $l / d$ and the last two entries give odd degenerate objects. Notice the even degenerate objects are isomorphic to the category of representations of the group $\left\langle x, y \mid x^{2}=y^{2}=(x y)^{d}=1\right\rangle$.

The case $D_{l}$ with $\left\{0,2 \lambda_{1}, \lambda_{j}, \lambda_{2 j}, \ldots, \lambda_{(n-1) j / 2}\right\}$ where $2 l=n j$ for $n$ odd. Again

$$
\begin{aligned}
& C_{2 \lambda_{1}}=e^{\pi i(4+2 \cdot 2 \cdot(2 l-2) / 2) /(2 l)}=1, \\
& C_{\lambda_{m j}}=e^{\pi i(m j+2 m j(2 l-m j-1) / 2) /(2 l)}=e^{\pi i\left(m j-m^{2} j^{2} /(2 l)\right)},
\end{aligned}
$$

so $2 \lambda_{1}$ is still even degenerate and

$$
\begin{aligned}
C_{\lambda_{(m \pm p) j}} C_{\lambda_{p j}}^{-1} C_{\lambda_{m j}}^{-1} & =e^{\mp \pi i m p j^{2} / l} \\
C_{\lambda_{l-(m+p) j}} C_{\lambda_{m j}}^{-1} C_{\lambda_{p j}}^{-1} & =e^{-\pi i m p j^{2} / l}, \\
C_{2 \lambda_{1}} C_{\lambda_{m j}}^{-1} C_{\lambda_{m j}}^{-1}=C_{0} C_{\lambda_{m j}}^{-1} C_{\lambda_{m j}}^{-1} & =e^{-\pi i m^{2} j^{2} / l} .
\end{aligned}
$$

This will be 1 for all $p$, and thus $\lambda_{m j}$ will be degenerate, exactly when $m$ is a multiple of $n / d$, where $d$ is the greatest common divisor of $n$ and $j$. If $m=r n / d$, 
then

$$
C_{\lambda_{m j}}=e^{\pi i\left(2 r l / d-2 r^{2} l / d^{2}\right)}
$$

which is always 1 . Thus the set of simple degenerate objects is

$$
\left\{0,2 \lambda_{1}, \lambda_{l / d}, \lambda_{2 l / d}, \ldots, \lambda_{(d-1) l / d}\right\},
$$

all degenerate objects are even, and again the category of even degenerate objects is isomorphic to the representation category of the group $\left\langle x, y \mid x^{2}=y^{2}=(x y)^{d}=1\right\rangle$.

\section{Tensor closed implies closed}

The definition of closed involves two conditions: closed under the truncated tensor product and closed under duality. Under the sort of conditions found in the quantum group examples the first condition actually implies the second. In particular for any sum of weights in the Weyl alcove, the set of weights appearing as summands of tensor powers of that sum is one of the closed subsets classified above. This is of particular relevance to skein-theoretic and Young diagrammatic approaches to the link invariants (see, e.g., [Turaev and Wenzl 1993]), where all the link information is recovered from cabling — that is, tensor powers — of an invariant corresponding to one particular weight, corresponding to the fundamental representation. The approach to the proof of the following proposition was suggested to the author by A. Liakhovskaia.

Proposition. If $\lambda$ is in the Weyl alcove then there is an $n$ such that $\lambda^{\otimes n}$ contains $\lambda^{\dagger}$ as a summand.

Proof. We shall actually show there is an $m$ such that $\lambda^{\otimes m}$ contains the weight 0 as a summand: Of course $n=m-1$ then suffices for the proposition.

For any two elements of the Weyl alcove $\lambda$ and $\gamma$ let $S_{\lambda, \gamma}$ be the value of the link invariant on the Hopf link with its two components labeled by $\lambda$ and $\gamma$ respectively. Recall from [Sawin 2002b] that viewed as a $\left|\Lambda_{0}\right|$-by- $\left|\Lambda_{0}\right|$ matrix $S$ is nondegenerate, and that

$$
\sum_{\gamma \in \Lambda_{0}} \mathrm{qdim}(\gamma) S_{\lambda, \gamma}=\delta_{\lambda, 0} \sum_{\gamma \in \Lambda_{0}} \mathrm{qdim}(\gamma)^{2}
$$

Thus if $\sum_{\gamma} S_{\lambda \otimes m}, \gamma$ is nonzero $\lambda^{\otimes m}$ contains 0 as a summand. Thus it suffices to show $\sum_{\gamma} S_{\lambda \otimes m}, \gamma$ is nonzero for some $m$. Dividing by $\operatorname{qdim}(\lambda)^{m}$ we see $(5-1)$

$$
\sum_{\gamma} S_{\lambda \otimes m, \gamma} \operatorname{qdim}(\gamma) / \mathrm{qdim}(\lambda)^{m}=\sum_{\gamma}\left[S_{\lambda, \gamma} / \mathrm{qdim}(\gamma) \mathrm{qdim}(\lambda)\right]^{m} \mathrm{qdim}(\gamma)^{2} .
$$

Now

$$
S_{\lambda, \gamma}=\sum_{\mu \in \Lambda_{0}} N_{\lambda, \gamma}^{\mu} C_{\mu} C_{\lambda}^{-1} C_{\gamma}^{-1} \mathrm{qdim}(\mu)
$$


and

$$
S_{\lambda \otimes \gamma, \mu}=S_{\lambda, \mu} S_{\gamma, \mu} / \mathrm{qdim}(\mu)
$$

so since $\operatorname{qdim}(\gamma)$ is positive, $C_{\mu}$ is a root of unity and

$$
\operatorname{qdim}(\lambda) \operatorname{qdim}(\gamma)=\sum_{\mu \in \Lambda_{0}} N_{\lambda, \gamma}^{\mu} \mathrm{qdim}(\mu),
$$

we see that $\left|S_{\lambda, \gamma} /(\operatorname{qdim}(\lambda) \mathrm{qdim}(\gamma))\right| \leq 1$, and $S_{\lambda, \gamma} /(\operatorname{qdim}(\lambda) \mathrm{qdim}(\gamma))$ is a root of unity when the absolute value equals one.

The quantity in brackets on the right-hand side of Equation (5-1) has modulus at most 1 for all values of $\gamma$, and for at least one term in the sum $(\gamma=0)$ has modulus equal to 1 . So for very large $m$ this sum is dominated by terms where the modulus is equal to 1 . In each of these terms the quantity $S_{\lambda, \gamma} / \operatorname{qdim}(\gamma) \operatorname{qdim}(\lambda)$ is a root of unity, so for infinitely many values of $m$ the value of $\left(S_{\lambda, \gamma} / \operatorname{qdim}(\gamma) \operatorname{qdim}(\lambda)\right)^{m}$ is equal to 1 simultaneously for all of the values of $\gamma$ for which the ratio has modulus 1 . Thus for sufficiently large $m$ the sum must be positive.

\section{Acknowledgments}

I thank I. Frenkel and A. Liakhovskaia for helpful conversations and suggestions.

\section{References}

[Andersen and Paradowski 1995] H. H. Andersen and J. Paradowski, "Fusion categories arising from semisimple Lie algebras", Comm. Math. Phys. 169:3 (1995), 563-588. MR 96e:17026 Zbl Zbl 0827.17010

[Bruguières 2000] A. Bruguières, "Catégories prémodulaires, modularisations et invariants des variétés de dimension 3", Math. Ann. 316:2 (2000), 215-236. MR 2001d:18009 Zbl 0943.18004

[Dijkgraaf and Witten 1990] R. Dijkgraaf and E. Witten, "Topological gauge theories and group cohomology", Comm. Math. Phys. 129:2 (1990), 393-429. MR 91g:81133 Zbl 0703.58011

[Humphreys 1972] J. E. Humphreys, Introduction to Lie algebras and representation theory, Graduate Texts in Mathematics 9, Springer, New York, 1972. MR 48 \#2197 Zbl 0254.17004

[Kassel 1995] C. Kassel, Quantum groups, Graduate Texts in Mathematics 155, Springer, New York, 1995. MR 96e:17041 Zbl 0808.17003

[Kirillov 1996] A. A. Kirillov, Jr., "On an inner product in modular tensor categories", J. Amer. Math. Soc. 9:4 (1996), 1135-1169. MR 97f:18007 Zbl 0861.05065

[Müger 2000] M. Müger, "Galois theory for braided tensor categories and the modular closure", Adv. Math. 150:2 (2000), 151-201. MR 2001a:18008 Zbl 0945.18006

[Murakami et al. 1992] H. Murakami, T. Ohtsuki, and M. Okada, "Invariants of three-manifolds derived from linking matrices of framed links", Osaka J. Math. 29:3 (1992), 545-572. MR 93h:57013 Zbl 0776.57009

[Sawin 2002a] S. F. Sawin, "Invariants of Spin three-manifolds from Chern-Simons theory and finite-dimensional Hopf algebras”, Adv. Math. 165:1 (2002), 35-70. MR 2003f:57028 Zbl 0994. 57011 
[Sawin 2002b] S. F. Sawin, "Jones-Witten invariants for nonsimply connected Lie groups and the geometry of the Weyl alcove", Adv. Math. 165:1 (2002), 1-34. MR 2003d:57055 Zbl 0997.57043 [Sawin 2006] S. Sawin, "Quantum groups at roots of unity and modularity”, 2006.

[Turaev 1994] V. G. Turaev, Quantum invariants of knots and 3-manifolds, Studies in Mathematics 18, de Gruyter, Berlin, 1994. MR 95k:57014 Zbl 0812.57003

[Turaev and Wenzl 1993] V. Turaev and H. Wenzl, "Quantum invariants of 3-manifolds associated with classical simple Lie algebras", Internat. J. Math. 4:2 (1993), 323-358. MR 94i:57019 Zbl 0784.57007

[Wenzl 1993] H. Wenzl, "Braids and invariants of 3-manifolds", Invent. Math. 114:2 (1993), 235275. MR 94i:57021 Zbl 0804.57007

Received October 15, 2004.

STEPHEN F. SAWIN

BNW 105

FAIRFIELD UNIVERSITY

1078 N. BENSON ROAD

FAIRFIELD CT 06824

United StATES

sawin@cs.fairfield.edu

cs.fairfield.edu/ sawin 